\title{
Coupled three-state oscillators
}

\author{
T. Prager ${ }^{1}$, B. Naundorf ${ }^{2}$, and L. Schimansky-Geier ${ }^{1}$ \\ ${ }^{1}$ Institute of Physics, Humboldt-University of Berlin, Invalidenstr. 110, 10115 \\ Berlin, Germany \\ ${ }^{2}$ Max-Planck-Institut für Strömungsforschung and Institut für Nichtlineare \\ Dynamik der Universität Göttingen, Bunsenstr. 10, 37073 Göttingen, Germany
}

\begin{abstract}
We investigate globally coupled stochastic three-state oscillators, which we consider as general models of stochastic excitable systems. We compare two situations:in the first case the transitions between the three states of each unit $1 \rightarrow 2 \rightarrow 3 \rightarrow 1$ are determined by Poissonian waiting time distributions. In the second case only transition $1 \rightarrow 2$ is Poissonian whereas the others are deterministic with a fixed delay. When coupled the second system shows coherent oscillations whereas the first remains in a stable stationary state. We show that the coherent oscillations are due to a Hopf-bifurcation in the dynamics of the occupation probabilities of the discrete states and discuss the bifurcation diagram.
\end{abstract}

\section{Introduction}

Coupled dynamic units are of great importance to understand cooperative behavior in deterministic dynamical systems. Many investigations on different levels of description like reaction diffusion equations, discrete deterministic maps, globally connected (mean field) or nonlocal models etc. give nowadays a good understanding of temporal and spatial pattern formation in different fields of science [1].

In the case of a coupled stochastic dynamics, stochastic source terms have to be incorporated in the deterministic dynamics. From simulations and equations for the moments it was shown that noise may be the origin of nontrivial behavior leading to noise induced order at non-vanishing noise intensities [2]. In particular, a new type of non-equilibrium phase transitions was found and it was shown that the transmission of signals even benefits from the presence of noise [3]. 
Principal insight of noise induced behavior has been achieved by introducing stochastic coupled units with few discrete states. For instance, stochastic resonance became a useful application in many different contexts after the formulation of two-state stochastic systems with periodically driven rates [4]. Also coupled chains have explained front motion in fluctuating media and array enhanced stochastic resonance [5]. Noise induced fluxes in sawtooth like potentials and media have also been considered with such stochastic systems incorporating a small number of possible states [6].

Most of the present simple discrete systems consider Poissonian transitions between the $n$ states of a unit. But such approximation, yielding a Markovian description, require often the introduction of a large number of variables or states. Whereas random walks are often considered with different waiting time distributions [7] investigations of stochastic discrete dynamical units with, for example, delayed feedback, has started only recently $[8,9]$.

In [10] we presented the occurrence of global oscillations in coupled three state oscillators with fixed waiting time [9] as a prototypical model for coupled excitable units. Here we compare this model with its Poissonian counterpart. We start from a common description for stochastic three state dynamics with general waiting time distributions. Whereas the resulting local dynamics characterized by its stationary density and power spectrum behaves similarly in both cases, the coupled cases exhibit a different behavior. In the globally coupled network with Poissonian transitions only stationary states can be encountered due to the high randomness of the transitions. Contrary, the delayed network shows coherent oscillations [11] and synchronization is found, as observed before in numerical investigations of excitable systems [12].

\section{Single three state units}

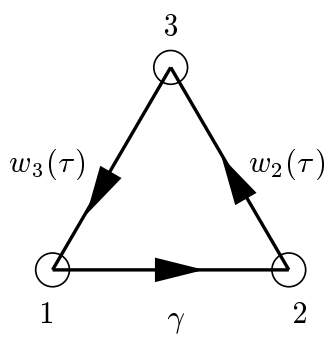

Fig. 1. A single stochastic three-state unit. The process $1 \rightarrow 2$ is Poissonian, while the transitions $2 \rightarrow 3$ and $3 \rightarrow 1$ are are governed by the waiting time distribution $w_{2}(\tau)$ and $w_{3}(\tau)$.

Our prototypical model of an excitable system is shown in Fig. 1. The unit aims to mimic a single stochastic excitable system. The transition from state $1 \rightarrow 2$ is the excitation step and occurs with rate $\gamma$. The transitions from $2 \rightarrow 3$ and from $3 \rightarrow 1$ model the return and take place with arbitrary waiting 
time distributions $w_{2}(\tau)$ and $w_{3}(\tau)$, respectively. As output of the unit we assign $f(t)=1$ if the unit is in state 3 and $f(t)=0$, otherwise.

First we derive equations which govern the dynamics of the probabilities $P_{i}(t)$, $i=1,2,3$, that the system is in state $i$ at time $t$. To this end we introduce the probability densities $P_{i}(t, \tau)$ that the system is in state $i$ at time $t$ and has entered it at time $t-\tau$. The relation between these probability densities and the probabilities $P_{i}(t)$ is given by $P_{i}(t)=\int_{0}^{\infty} d \tau P_{i}(t, \tau)$. The flow $J_{i}(t)$ of probability between state $i$ and $i+1$ can be expressed in terms of the probability densities $P_{i}(t, \tau)$ and the waiting time distributions $w_{i}(\tau)$. If this transition is governed by a Poisson process with rate $\gamma$ the flow is just this rate times the probability that the particle is in state $i$. Thus, for the transition $1 \rightarrow 2 J_{1}(t)=\gamma P_{1}(t)$. The probability density $P_{2}(t, \tau)$ is the flow $J_{1}$ at time $t-\tau$ times the probability that the process stays in state 2 at least the time $\tau$. The latter is determined by $w_{2}(t)$. In summary,

$$
P_{2}(t, \tau)=\gamma P_{1}(t-\tau)\left[1-\int_{0}^{\tau} d \tau^{\prime} w_{2}\left(\tau^{\prime}\right)\right]
$$

By definition we immediately obtain

$$
P_{2}(t)=\int_{0}^{\infty} d \tau \gamma P_{1}(t-\tau)\left[1-\int_{0}^{\tau} d \tau^{\prime} w_{2}\left(\tau^{\prime}\right)\right]
$$

In case of a general waiting time distribution we introduce a waiting time dependent conditioned rate $\phi_{i}(\tau)$ from the relation

$$
w_{i}(\tau)=\phi_{i}(\tau)\left[1-\int_{0}^{\tau} d \tau^{\prime} w_{i}\left(\tau^{\prime}\right)\right]
$$

It gives the rate of escaping from state $i$ after having waited the time $\tau$ and determines the flow between $i$ and $i+1$ according to $J_{i}(t)=\int_{0}^{\infty} d \tau \phi_{i}(\tau) P_{i}(t, \tau)$. Subsuming we obtain

$$
P_{i+1}(t)=\int_{0}^{\infty} d \tau J_{i}(t-\tau)\left[1-\int_{0}^{\tau} d \tau^{\prime} w_{i+1}\left(\tau^{\prime}\right)\right] .
$$

Inserting $P_{2}(t, \tau)$ from eq.( 1$)$ into this equation for $i=2$ finally leads to

$$
P_{3}(t)=\int_{0}^{\infty} d \tau \int_{0}^{\infty} d \tau^{\prime} \gamma P_{1}\left(t-\tau-\tau^{\prime}\right) w_{2}\left(\tau^{\prime}\right)\left[1-\int_{0}^{\tau} d \tau^{\prime \prime} w_{3}\left(\tau^{\prime \prime}\right)\right]
$$


The system of eqs. (2) and (5) is completed and becomes closed by the normalization condition

$$
P_{1}(t)=1-P_{2}(t)-P_{3}(t)
$$

Because of the first Poissonian transition the system is generally ergodic and possesses one stationary asymptotic solution.

Further information of the oscillator can be extracted from its spectral properties. To this end we consider the stochastic process (output) $f(t)$ as introduced, previously. This process corresponds to a sequence of pulses whose lengths $\rho$ are distributed according to $w_{\rho}(t)=w_{3}(t)$ and the intervals $\sigma$ between two consequent pulses are distributed according to $w_{\sigma}(t)=\int_{0}^{t} d \tau w_{1}(\tau) w_{2}(t-\tau)=$ $\gamma \int_{0}^{t} d \tau \exp (-\gamma \tau) w_{2}(t-\tau)$. The spectrum of $z(t)-\langle z(t)\rangle$ can be calculated employing renewal theory [13] and is given by

$$
S(\omega)=\frac{4}{\omega^{2}(\langle\rho\rangle+\langle\sigma\rangle)} \operatorname{Re} \frac{\left(1-\Theta_{\rho}(\omega)\right)\left(1-\Theta_{\sigma}(\omega)\right)}{1-\Theta_{\rho}(\omega) \Theta_{\sigma}(\omega)}
$$

where $\Theta_{\rho}(\omega):=\langle\exp (i \omega \rho)\rangle$ and $\Theta_{\sigma}(\omega):=\langle\exp (i \omega \sigma)\rangle$ are the characteristic functions of the random variables $\rho$ and $\sigma$ respectively.

Let us now consider the case where all three transitions are governed by Poisson processes, i.e. $w_{2}(\tau)=\gamma_{2} \exp \left(-\gamma_{2} \tau\right)$ and $w_{3}(\tau)=\gamma_{3} \exp \left(-\gamma_{3} \tau\right)$. After taking the derivative of eq. (2) with respect to $t$ we obtain

$$
\dot{P}_{2}(t)=-\int_{0}^{\infty} d \tau\left[\frac{\partial}{\partial \tau} \gamma P_{1}(t-\tau)\right] \exp \left(-\gamma_{2} \tau\right)=-\gamma_{2} P_{2}(t)+\gamma P_{1}(t)
$$

A similar calculation can be done for $P_{3}(t)$, leading to

$$
\dot{P}_{3}(t)=-\gamma_{3} P_{3}(t)+\gamma_{2} P_{2}(t)
$$

i.e. one retrieves the expected rate dynamics. To determine the corresponding spectrum as defined in the previous section we use $w_{\rho}(t)=\gamma_{3} \exp \left(-\gamma_{3} \rho\right)$ and $w_{\sigma}(t)=\frac{\gamma \gamma_{2}}{\gamma-\gamma_{2}}\left(\exp \left(-\gamma_{2} t\right)-\exp (\gamma t)\right)$ which gives (see Fig. (2))

$$
S(\omega)=\frac{4\left(\left(\gamma+\gamma_{2}\right)^{2}+\omega^{2}\right)}{\left(\frac{1}{\gamma}+\frac{1}{\gamma_{2}}+\frac{1}{\gamma_{3}}\right)\left(\left(\gamma \gamma_{2}+\gamma_{2} \gamma_{3}+\gamma_{3} \gamma\right)^{2}+\omega^{2}\left(\gamma^{2}+\gamma_{2}^{2}+\gamma_{3}^{2}\right)+\omega^{4}\right)}
$$

Instead of a fixed rate for the transition $2 \rightarrow 3$ and $3 \rightarrow 1$ we now deal with the case of a fixed waiting time. The corresponding waiting time distributions 
are given by $w_{2}(\tau)=\delta\left(\tau-\tau_{2}\right)$ and $w_{3}(\tau)=\delta\left(\tau-\tau_{3}\right)$. Inserting these into eqs. (5) and (2) leads to

$$
P_{2}(t)=\int_{0}^{\tau_{2}} d \tau \gamma P_{1}(t-\tau), \quad P_{3}(t)=\int_{0}^{\tau_{3}} d \tau \gamma P_{1}\left(t-\tau-\tau_{2}\right)
$$

supplemented by normalization, eq. (6). The calculation of the power spectrum is done analogously to the Markovian case, finally giving

$$
S(\omega)=\frac{2 \cos \left(\omega \tau_{3}\right)-2}{\left(\frac{1}{\gamma}+\tau_{2}+\tau_{3}\right)\left(\omega^{2}+\gamma^{2}\right)\left(1+\frac{\gamma}{\sqrt{\omega^{2}+\gamma^{2}}} \sin \left(\left(\tau_{2}+\tau_{3}\right) \omega+\phi\right)\right)}, \quad \tan \phi=\frac{\omega}{\gamma} .
$$

With $\tau_{2}$ fixed to 0.3 and $\tau_{3}$ fixed to 0.7 the spectra for different values of the rate $\gamma$ are presented in figure 2 .
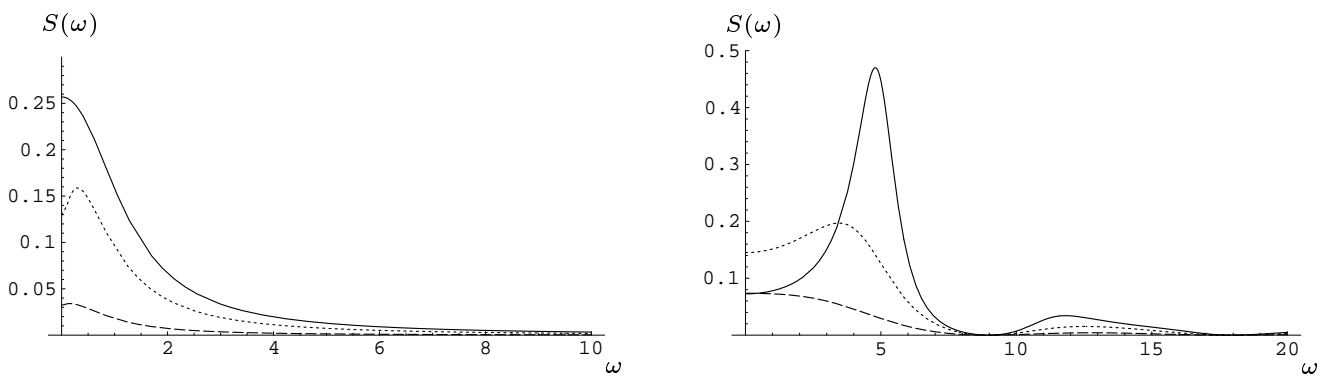

Fig. 2. The power spectrum for a single stochastic oscillator which Poissonian (left) and deterministic waiting time distributions (right). Left: $\gamma=0.1, \gamma_{3}=1$. Solid: $\gamma_{2}=1$, dotted: $\gamma_{2}=0.1$. dashed: $\gamma_{2}=0.01$. Right: $\tau_{2}=0.3$ and $\tau_{3}=0.7$. Solid: $\gamma=2.0$, dotted: $\gamma=0.5$, dashed: $\gamma=0.1$.

The spectra do not differ too much from the Poissonian case. In both cases it is possible to have a maximum in the spectrum at frequencies different from zero.

\section{Globally coupled three state units}

We now consider a set of $N$ three state oscillators as described in the previous section. A global coupling between the single units is introduced by letting the rate $\gamma$, which governs the transition $1 \rightarrow 2$, depend on the common output

$$
f(t)=\frac{1}{N} \sum_{i=1}^{N} f_{i}(t)
$$


where $f_{i}(t)$ is now the output of a single unit.

In the limit $N \rightarrow \infty$ the state of the total system can be described by the ensemble averaged occupation probabilities $P_{i}(t)$ that a single oscillator is in state $i$ at time $t$. Since $f(t)$ converges to the occupation probability of the third state, $\gamma$ becomes a function of $P_{3}(t)$.

Equations which govern the dynamics of the ensemble averaged occupation probabilities are derived in the same manner as eqs. (5) and (2) except that one has to take care that the rate $\gamma$ is no longer a constant but is dependent on $P_{3}(t)$. One eventually arrives at

$$
\begin{aligned}
& P_{2}(t)=\int_{0}^{\infty} d \tau \gamma\left(P_{3}(t-\tau)\right) P_{1}(t-\tau)\left[1-\int_{0}^{\tau} d \tau^{\prime} w_{2}\left(\tau^{\prime}\right)\right] \\
& P_{3}(t)=\int_{0}^{\infty} d \tau \int_{0}^{\infty} d \tau^{\prime} \gamma\left(P_{3}\left(t-\tau-\tau^{\prime}\right)\right) P_{1}\left(t-\tau-\tau^{\prime}\right) w_{2}\left(\tau^{\prime}\right)\left[1-\int_{0}^{\tau} d \tau^{\prime \prime} w_{3}\left(\tau^{\prime \prime}\right)\right]
\end{aligned}
$$

and normalization, eq.(6). These equations are now nonlinear in the occupation probabilities due to the dependency of $\gamma$ on the state of the system.

The system dynamics of the coupled system can be understood by analyzing the stationary solutions and its stability properties. The stationary solutions $P_{1}^{*}, P_{2}^{*}$ and $P_{3}^{*}$ are defined by setting $P_{i}(t)=$ const. in eq. (9). Using $\int_{0}^{\infty}(1-$ $\int_{0}^{\tau} d \tau^{\prime} w_{2 / 3}\left(\tau^{\prime}\right) d \tau=\int_{0}^{\infty} d \tau \tau w_{2 / 3}(\tau)=: \bar{w}_{2 / 3}$, which is the mean waiting time in state 2 or 3 , respectively, we obtain implicitly

$$
P_{1}^{*}=\frac{\gamma^{-1}\left(P_{3}^{*}\right)}{T}, \quad P_{2}^{*}=\frac{\bar{w}_{2}}{T}, \quad P_{3}^{*}=\frac{\bar{w}_{3}}{T}
$$

with $T=\gamma^{-1}\left(P_{3}^{*}\right)+\bar{w}_{2}+\bar{w}_{3}$ being the mean time of one cycle. In contrast to the uncoupled case, where exactly one asymptotic stationary solution exists, the asymptotic behavior may now be more complex due to the nonlinearity of eqs. (9). Depending on the chosen coupling function there can be several not necessarily stable stationary solutions.

The local stability of a stationary solution can be derived by inserting the Ansatz $P_{i}(t)=P_{i}^{*}+A_{i} \exp (\lambda t), \lambda \neq 0$, into the linearized dynamical equations. Therefrom we obtain the characteristic equation

$$
0=1+\gamma\left(P_{3}^{*}\right) \int_{0}^{\infty} d \tau e^{-\lambda \tau}\left(1-\int_{0}^{\tau} d \tau^{\prime} w_{2}\left(\tau^{\prime}\right)\right)
$$




$$
+\left(\gamma\left(P_{3}^{*}\right)-\gamma^{\prime}\left(P_{3}^{*}\right) P_{1}^{*}\right) \int_{0}^{\infty} d \tau\left[e^{-\lambda \tau}\left(1-\int_{0}^{\tau} d \tau^{\prime} w_{3}\left(\tau^{\prime}\right)\right)\right] \int_{0}^{\infty} d \tau e^{-\lambda \tau} w_{2}(\tau)
$$

In the case with Poissonian transitions the mean waiting times in state 2 and 3 are given by $\bar{w}_{2}=1 / \gamma_{2}$ and $\bar{w}_{3}=1 / \gamma_{3}$. There may be several stable and unstable stationary solutions depending on the chosen coupling function. Eq. (11) corresponds to the characteristic equation of a two dimensional dynamical system:

$$
\lambda^{2}+\lambda\left(\gamma\left(P_{3}^{*}\right)+\gamma_{2}+\gamma_{3}\right)+\gamma\left(P_{3}^{*}\right) \gamma_{2}+\gamma_{2} \gamma_{3}+\gamma_{3} \gamma\left(P_{3}^{*}\right)-\gamma_{2} \gamma^{\prime}\left(P_{3}^{*}\right) P_{1}^{*}=0
$$

In order to decide whether there exist limit cycles we calculate the divergence of the flow in the phase space which is $-\left(\gamma\left(P_{3}\right)+\gamma_{2}+\gamma_{3}\right)$. As the rates $\gamma\left(P_{3}\right)$, $\gamma_{2}$ and $\gamma_{3}$ are always positive the divergence is everywhere negative. Therefore no limit cycles exist, i.e. the system shows no coherent oscillations.

For fixed waiting times $\tau_{2}$ and $\tau_{3}$ the dynamics of the occupation probabilities is reduced to

$$
\begin{aligned}
& P_{2}(t)=\int_{0}^{\tau_{2}} d \tau \gamma\left(P_{3}(t-\tau)\right) P_{1}(t-\tau), \\
& P_{3}(t)=\int_{0}^{\tau_{3}} d \tau \gamma\left(P_{3}\left(t-\tau-\tau_{2}\right)\right) P_{1}\left(t-\tau-\tau_{2}\right), \quad P_{1}(t)=1-P_{2}(t)-P_{3}(t)
\end{aligned}
$$

The stationary solutions are given by eq. (10) with $\bar{w}_{2}=\tau_{2}$ and $\bar{w}_{3}=\tau_{3}$. The characteristic equation is easily derived from the general eq. (11) to be

$$
\gamma\left(P_{3}^{*}\right)\left(e^{-\lambda\left(\tau_{2}+\tau_{3}\right)}-1\right)-\gamma^{\prime}\left(P_{3}^{*}\right) P_{1}^{*}\left(e^{-\lambda\left(\tau_{2}+\tau_{3}\right)}-e^{-\lambda \tau_{2}}\right)-\lambda=0 .
$$

Such characteristic equations are typical for dynamical systems involving fixed delays [14].

\section{Sigmoidal coupling}

In the following we review the behavior of the coupled system for a sigmoidal rate function as presented in $[10]$

$$
\gamma\left(P_{3}(t)\right)=\gamma_{0}\left(1+\Delta \tanh \left[-\frac{f(t)-f^{*}}{2 \sigma}\right]\right)
$$


For this rate function, there exists exactly one stationary solution of the system dynamics. The coupling strength is mediated by $\sigma$ : The smaller $\sigma$ the stronger is the dependence of the rate on $P_{3}(t)$. For small $\sigma$ the rate changes rapidly from $\gamma_{1}:=\gamma_{0}(1+\Delta)$ to $\gamma_{2}:=\gamma_{0}(1-\Delta)$ if the value of $P_{3}(t)$ crosses $f_{0}$. For large values of $\sigma$ the rate is nearly independent of $P_{3}(t)$ and equal to $\gamma_{0}$.

In case of a Poissonian waiting time distribution this stationary solution is necessarily stable as shown in the last section. The high randomness in the transitions does not allow the excitation of oscillating behavior, i.e the coupled system does not differ substantially from the uncoupled case.

For fixed waiting times, however, the behavior changes due to the coupling. For each value of $\gamma_{0}$ there is a finite region in which the ensemble oscillates.

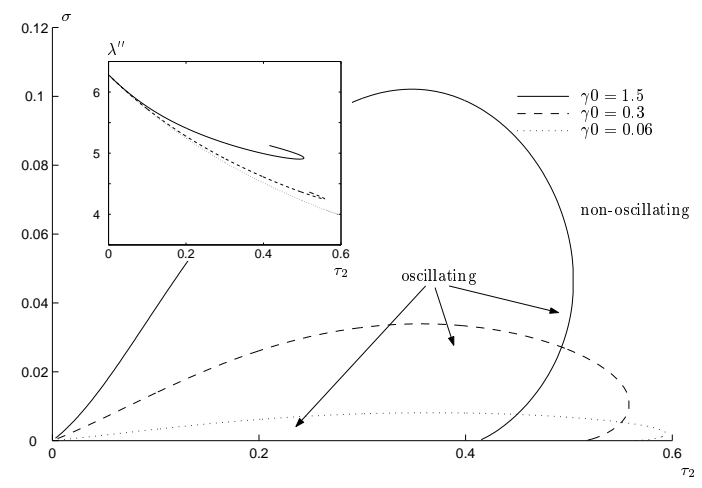

Fig. 3. Solutions of the characteristic equation with $\operatorname{Re} \lambda=0$ for different values of $\gamma_{0}$. The parameter $\Delta$ is fixed to $2 / 3, f_{0}$ is chosen to be the stationary solution $P_{3}^{*}$ at $\tau_{2}=0.3$, which is about $0.42,0.16$ and 0.04 respectively. The inset shows the corresponding frequency at the bifurcation.

This region grows with increasing values of $\gamma_{0}$. If $\sigma$ is chosen too large, coherent behavior cannot be observed. Interestingly, there exist values of $\tau_{2}$ where $\sigma$ has to exceed some finite value to trigger the oscillations. We have verified this transition between non-oscillating, oscillating and again non-oscillating behavior in numerical simulations.

One may ask, whether there is some connection between the oscillatory behavior of a single oscillator and the oscillatory behavior of the total system. To this end we have carried out simulations with the sigmoidal rate function, where $\gamma_{0}$ has been chosen to be $0.06,0.3$ and 1.5 respectively. In the first case the rate lies always in the non oscillating region of a single oscillator, in the second case, it switches between the oscillating and non oscillating region, and in the third case it is always in the oscillating region. $\sigma$ has been fixed to 0.001 and the values of $f_{0}$ are chosen to be approximately equal to the stationary solution $P_{3}^{*}$. The results are shown in figure 4 . In each case we observe coherent oscillations of the total system, although the amplitude of the oscillations depends strongly on the chosen rate. Interestingly however, the 

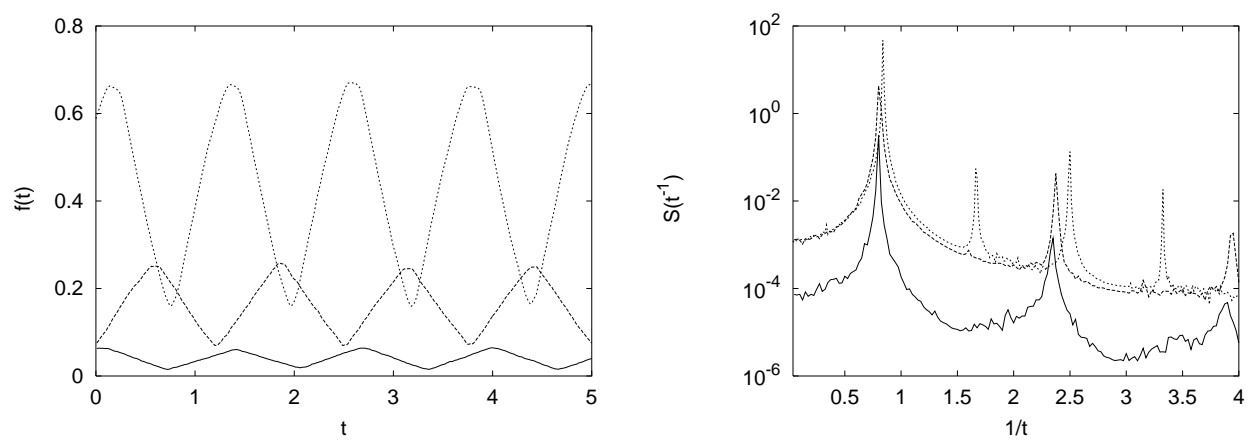

Fig. 4. Left: Behavior of the ensemble for different values $\gamma_{0}$ and $f_{0}$ with $\sigma$ fixed to 0.001 and $\Delta$ to $2 / 3$ and $\tau_{2}$ to 0.3. Solid: $\gamma_{0}=0.06, f_{0}=0.04$ Dashed: $\gamma_{0}=0.3, f_{0}=0.16$ Dotted: $\gamma_{0}=1.5, f_{0}=0.42$ The simulations have been performed for a system consisting of 10000 units. Right: The corresponding spectra of the common output $f(t)$

frequency of the oscillations is nearly independent on $\gamma_{0}$ although the mean time of one cycle of a single oscillator strongly varies with $\gamma_{0}$. Taking into account that the mean waiting time of a Poisson process with rate $\gamma$ is $1 / \gamma$ the mean time for one cycle of a single oscillator lies between $1+1 /\left(\gamma_{0}(1+\Delta)\right)$ and $1+1 /\left(\gamma_{0}(1-\Delta)\right)$. This corresponds to frequencies between approximately 0.02 and 0.09 for $\gamma_{0}=0.06,0.09$ and 0.3 for $\gamma_{0}=0.3$ and 0.3 and 0.7 for $\gamma_{0}=1.5$. Obviously the frequencies of the coherent oscillations one observes do not correspond to these frequencies, especially in the first case they are even of a different order of magnitude. However there is a good agreement between the observed frequencies and the frequencies at the bifurcation, which are shown in the inset of Fig. 3.

\section{Conclusion}

We have investigated stochastic three state oscillators, which we propose as a model for excitable systems driven by noise. First we considered the behavior of the single units and inspected their spectral properties for the case of Poissonian and deterministic transitions. It turns out that the process with deterministic transitions is a good simple model describing excitable dynamics with rich spectral behavior. When coupled globally both models behave quite differently. While in the Poissonian case there are no oscillating solutions, in the case with fixed waiting times the coupling leads to coherent oscillations of the network. A stability analysis reveals, that these oscillations are due to a Hopf bifurcation in the dynamics of the occupation probabilities. Interestingly, the global oscillations occur even for units which have maximal power at zero frequency. It is the common situation of noise induced phase transitions, that coupling might induce ordered behavior despite the fact that the constituting 
elements involve a lot of randomness $[2,3,5]$.

This work was supported by DFG-Sfb 555. We thank B. Lindner and M. Zaks for help and fruitful comments.

\section{References}

[1] A. S. Mikhailov, Foundations of Synergetics I(Springer, Berlin-Heidelberg-New York, 1990)

[2] C. van den Broeck, J. M. R. Parrondo, and R. Toral, Phys. Rev. Lett. 73, 3395 (1994); J. García-Ojalvo and J. M. Sancho,Noise in Spatially Extended Systems (Springer, New York, 1999).

[3] A. A. Zaikin, J. García-Ojalvo, L. Schimansky-Geier, and J. Kurths Phys. Rev. Lett. 88, 010601 (2002).

[4] B. McNamara and K. Wiesenfeld, Phys. Rev A 39, 4854 (1989).

[5] V. Anishchenko, et al. Chaotic and Stochastic Processes in Dynamic Systems( Springer, Berlin-Heidelberg-New York, 2002).

[6] R. D. Astumian, Science 276, 917 (1997); L. Schimansky-Geier, M. Kschischo, T. Fricke Phys. Rev. Lett. 79, 3335 (1997); J. A. Freund, and L. SchimanskyGeier Phys. Rev. E 60, 1304 -1309 (1999).

[7] G. H. Weiss, Aspects and Applications of the Random Walk (North-Holland, Amsterdam, 1994)

[8] L. S. Tsimring and A. Pikovsky Phys. Rev. Lett. 87, 250602 (2001); L. Q. Zhou, X. Jia, and Q. Ouyang, Phys. Rev. Lett. 88, 138301 (2002)

[9] A. Nikitin, Z. Néda, and T. Vicsek Phys. Rev. Lett. 87, 024101 (2001).

[10] B. Naundorf, T. Prager and L. Schimansky-Geier, submitted for publication

[11] A. Pikovsky, J. Kurths, Phys. Rev. Lett. 78, 775 (1997); B. Lindner and L. Schimansky-Geier, Phys. Rev E 61, 6103-6110 (2000).

[12] C. Kurrer and K. Schulten, Phys. Rev. E 51, 6213 (1995); H. Hempel, L. Schimansky-Geier, and J. Garcia-Ojalvo Phys. Rev. Lett. 82, 3713-3716 (1999); A. Neiman, L. Schimansky-Geier, A. Cornell-Bell, and F. Moss Phys. Rev. Lett. 83, 4896-4899 (1999).

[13] R. L. Stratonovich, Topics in the Theory of Random Noise I (Gordon and Breach, 1962) p. 175.; B. Lindner and L. Schimansky-Geier, Phys. Rev E 60 , 7270-7276 (1999).

[14] G. T. Gurija and M. A. Lifshits Z. Phys B 47, 71 (1982); L. Schimansky-Geier, Ch. Zülicke and E. Schöll, Physica A 188, 436 (1992). 